\title{
Electromagnetic interference shielding characteristics for orientation angle and number of plies of carbon fiber reinforced plastic
}

\author{
Hong Gun Kim ${ }^{1}$, Hee Jae Shin², Gwang-Cheol Kim³ , Hyung Joon Park ${ }^{4}$, Ho Joon Moon ${ }^{5}$ and Lee Ku Kwac,\$ \\ ${ }^{1}$ Department of Mechanical and Automotive Engineering, Jeonju University, Jeonju 560-759, Korea \\ ${ }^{2}$ Department of Mechanical Engineering, Jeonju University Graduate School, Jeonju 560-759, Korea \\ ${ }^{3}$ SG Tech Co., Ltd., Jeonju 560-759, Korea \\ ${ }^{4}$ Department of Carbon and Nano Engineering, Jeonju University Graduate School, Jeonju 560-759, Korea \\ ${ }^{5}$ NanoSolution Co., Ltd., Jeonju 561-844, Korea \\ ${ }^{6}$ Department of Carbon and Nano Engineering, Jeonju University, Jeonju 560-759, Korea
}

\section{Article Info}

Received 25 July 2014

Accepted 25 August 2014

*Corresponding Author

E-mail: kwac29@jj.ac.kr

Tel: $+82-63-220-3063$

Open Access

DOI: http://dx.doi.org/

10.5714/CL.2014.15.4.268

This is an Open Access article distributed under the terms of the Creative Commons Attribution Non-Commercial License (http://creativecommons.org/licenses/ by-nc/3.0/) which permits unrestricted non-commercial use, distribution, and reproduction in any medium, provided the original work is properly cited.

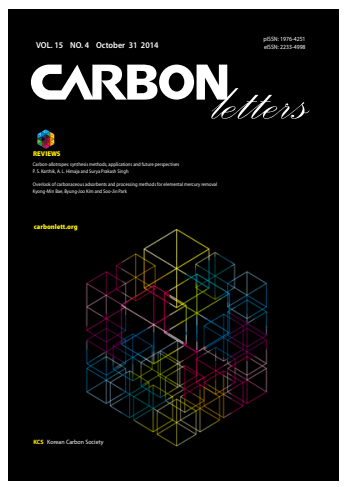

http://carbonlett.org

pISSN: $1976-4251$

eISSN: 2233-4998

Copyright $\odot$ Korean Carbon Society

\begin{abstract}
Recently, methods that usea carbon-based filler, a conductive nanomaterial, have been investigated to develop composite fillers cont7aining dielectric materials. In this study, we added geometric changes to a carbon fiber, a typical carbon-based filler material, by differentiating the orientation angle and the number of plies of the fiber. We also studied the electrical and electromagnetic shield characteristics. Based on the orientation angle of $0^{\circ}$, the orientation angle of the carbon fiber was changed between $0,15,30,45$, and $90^{\circ}$, and 2,4 , and 6 plies were stacked for each orientation angle. The maximum effect was found when the orientation angle was $90^{\circ}$, which was perpendicular to the electromagnetic wave flow, as compared to $0^{\circ}$, in which case the electrical resistance was small. Therefore, it is verified that the orientation angle has more of an effect on the electromagnetic interference shield performance than the number of plies.
\end{abstract}

Key words: carbon fiber reinforced plastic, composite, electromagnetic interference shield, orientation angle

\section{Introduction}

Recently, because of rapid industrialization and informatization, considerable attention has been paid to environmental pollution and its effects on the human body. From this rapid change, the problem of electromagnetic interference or compatibility (EMI/ EMC) caused by electronic devices and electric appliances has worsened because of the prevalent use of communication products such as mobile phones. The electromagnetic wave problem is not far away from our daily lives since it is closely related to modern life, in which TVs, PCs, and mobile phones are used. The human body directly exposed to these electromagnetic waves can face serious problems such as skin irritations; hallucinations; sleep disturbances; learning ability degradation; child growth distrurbances; various cancers such as lymphatic cancer, breast cancer, and brain tumors; myocardial infarction; infertility; birth defects; miscarriages; headaches; depression; and DNA damage. With respect to these problems, the World Health Organization (WHO) has published a considerable amount of literature. Further, many incidents of problems in the fields of operation and production have been reported because of the malfunctioning of or errors in devices throughout the industries, causing a serious safety problem for the operators. In the defense industry, it has also been reported that communication 
devices such as defense radars have malfunctioned. To shield this EMI, various methods have been used. In general, metalbased materials are used as EMI shield materials. However, recently, because of the increasing trend of lightweight devices, and from the viewpoint of energy reduction, many studies have been conducted on the use of polymeric materials, which are lighter than metal-based materials. However, in general, polymeric materials have no effect as an EMI shield. Therefore, in order for polymeric materials to exhibit a shielding performance, many studies have been conducted on materials that can effectively shield from EMI. These studies produce a plastic-reinforced composite that not only has good conductivity, but also absorbs electromagnetic waves because of thecoating of a transition metal such as $\mathrm{Cu}$ and $\mathrm{Ag}$. However, although using this transition metal can improve the electromagnetic shield performance, when such metal is used, there are problems of high price and oxidation of the metal. Hence, the use of this metal is not preferred [1-9].

Recently, in order to develop composite fillers containing dielectric materials, a method that uses a carbon-based filler, which is a conductive nanomaterial, was investigated. Kim and Kim [10] attempted to determine the characteristics of electromagnetic waves by utilizing the composite laminates of carbon nanotubes. Han et al. [11] found the characteristics of effective EMI shielding with respect to aluminum films, conductive fabric fibers, and nano-carbon black composites. Hong et al. [12] discovered the characteristics of electromagnetic waves by coating nickel on carbon fibers. As described above, most studies on EMI shield materials using carbonbased fillers have concentrated on the inherent characteristics of the material, but few studies have been conducted on the shielding effect by adding geometric changes to the shielding materials. In this study, the method of adding geometric changes to a carbon fiber, which is a typical carbon-based filler, was used to differentiate the orientation angle and the number of plies. Moreover, the related electrical and electromagnetic shield characteristics were studied.

\section{Measurement of EMI}

The fabric fiber used as reinforced material in this study was T300 fabric fiber CO6644B (weight, $300 \mathrm{~g} / \mathrm{m}^{2}$ ) manufactured by TORAYCA ${ }^{\circledR}$, and the epoxy resin used as the matrix was YD128 (e.e.w., 184-190 g/eq, viscosity: 11 500-13 $500 \mathrm{cps}$ ) based on the diglycidylether of bisphenol-A (DGEBA), which is a bifunctional epoxy oligomer manufactured by Kukdo Chemical Co. Ltd. As a hardener, 4,4'-diaminodiphenylmethane (DDM), a product of TCI, was used, and methylethylketone (MEK) was used as a diluent to lower the high viscosity of YD-128. The chemical structure of the epoxy resin and the hardener used in this study is shown in Fig. 1 [12].

The epoxy resin and the hardener DDM were mixed in a $1: 1$ equivalence ratio and melted at $70^{\circ} \mathrm{C}$, and $\mathrm{MEK}$ (weight ratio, 1:1) with epoxy resin was added to the dilute solution. The carbon fiber was impregnated with epoxy resin, and then a unidirectional carbon fabric prepreg was produced. Then, as shown in Fig. 2, based on an orientation angle of $0^{\circ}$, the orientation angle of the carbon fiber was changed to $0,15,30$,

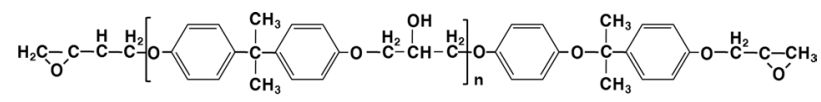

Diglycidylether of bisphenol-A (DGEBA, YD-128)<smiles>Nc1ccc(Cc2ccc(N)cc2)cc1</smiles>

Fig. 1. Chemical structure of DGEBA diaminodiphenylmethane.

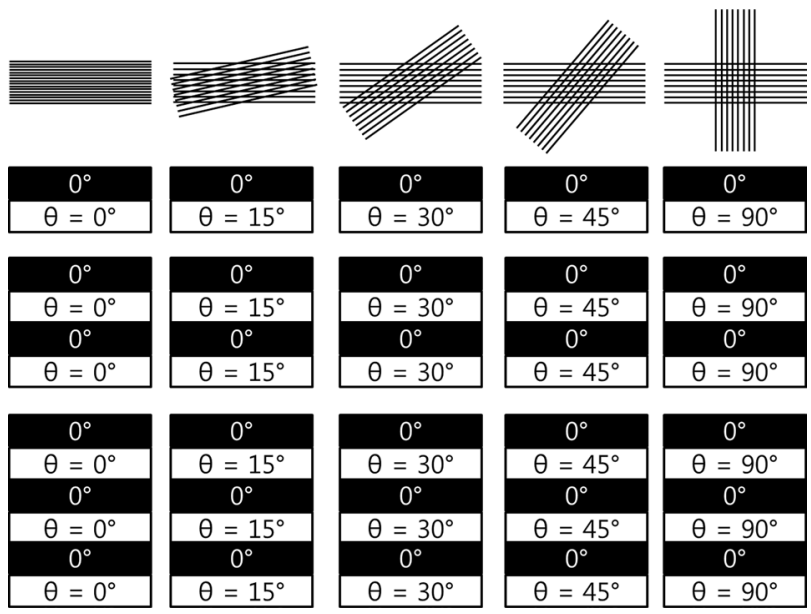

Fig. 2. Orientation angle and plies of unidirectional carbon fiber specimen.

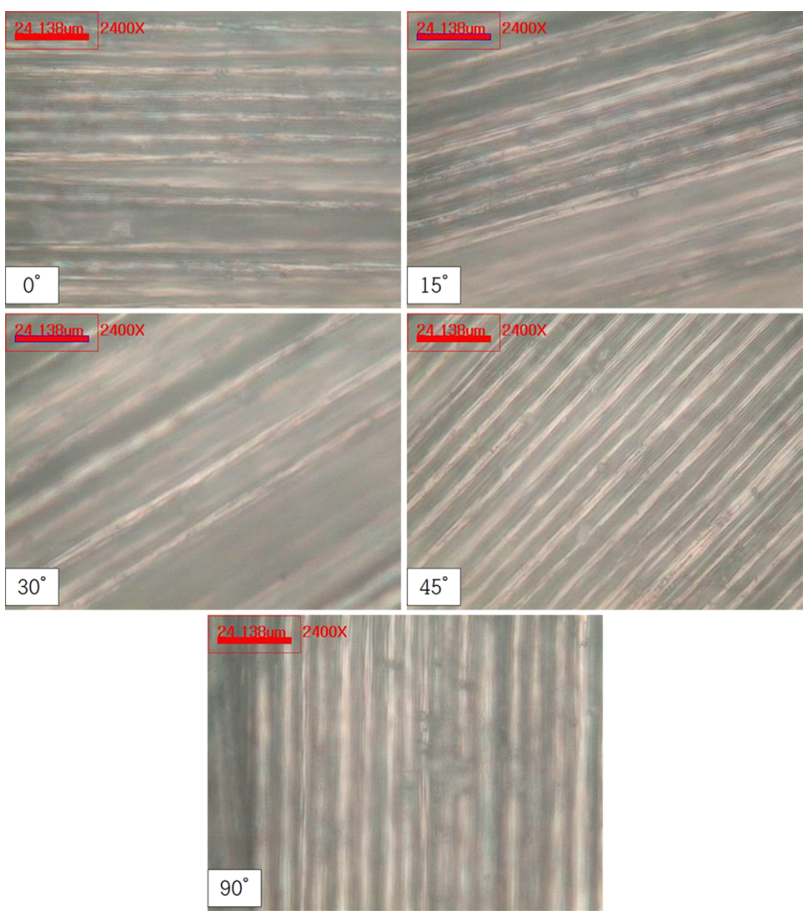

Fig. 3. Optical microscope image of the orientation angle of the carbon fiber.

45 , and $90^{\circ}$, and 2,4 , and 6 plies were stacked for each orientation angle. After this, the plies were hardened by vacuum bag molding for $180 \mathrm{~min}$ in order to produce carbon fiber re- 

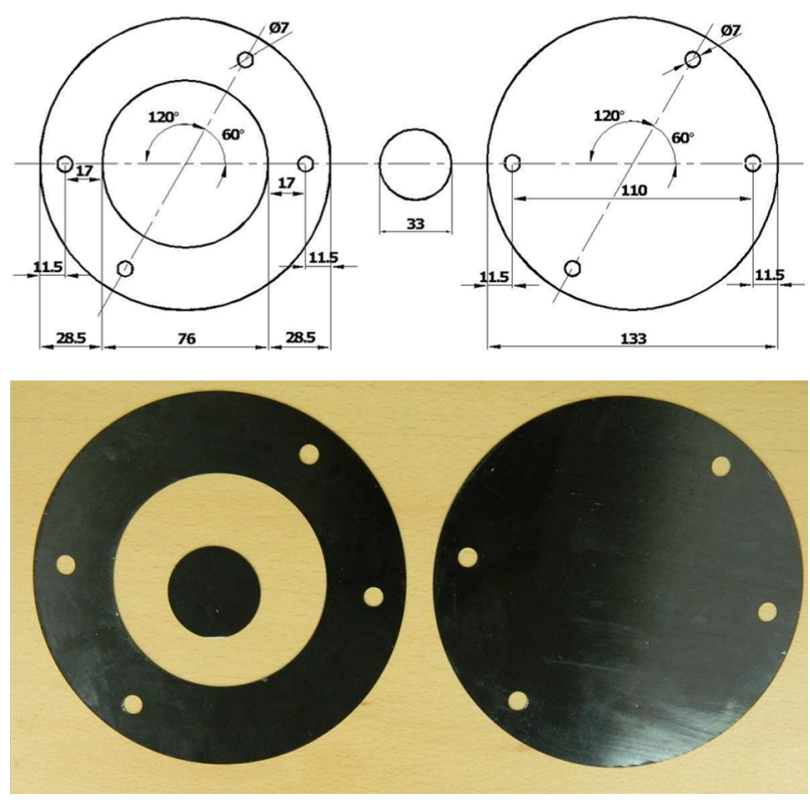

Fig. 4. Specimen dimensions and photograph of carbon fiber reinforced plastic for electromagnetic interference shielding. (a) Dimensions of specimen, (b) photograph of specimen.

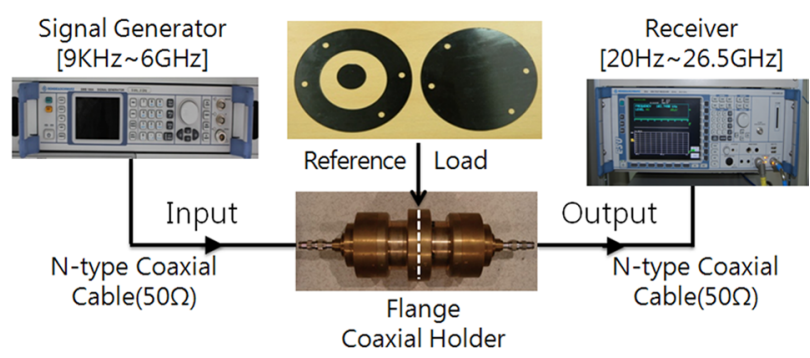

Fig. 5. Experimental configuration of electromagnetic interference shielding experiments.

inforced plastic (CFRP). Fig. 3 shows the optical microscope image based on the orientation angle.

The CFRP produced, as shown in Fig. 2, was modified to be a specimen having the dimensions of ASTM D4935-10, as shown in Fig. 4a, which produced a specimen shown in Fig. 4b. Further, as shown in Fig. 5, the electromagnetic strength was measured directly through the specimen materials using a flange coaxial transmission line fixture. This was in order to measure the EMI shield efficiency. Asystem was also configured using an electromagnetic test receiver and a signal generator.

The concept of an EMI shield is to absorb or reflect the waves through desired materials such that the external waves shall not be transmitted into an indoor environment or system. The shield capability can be defined by the extent to which it can attenuate an electromagnetic wave introduced into an object.

$$
S E(d B)=10 \log \left(\frac{P_{T}}{p_{R}}\right)
$$
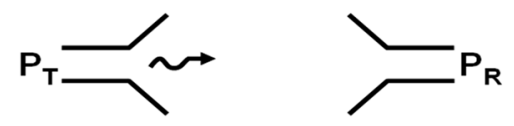

(a)
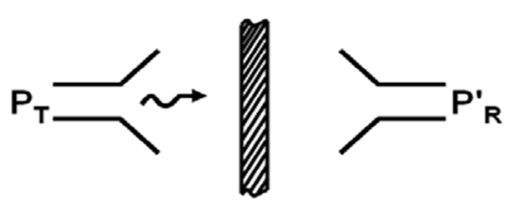

(b)

Fig. 6. Typical arrangement for measuring effectiveness of materials. (a) Unloaded specimen, (b) loaded specimen.

Considering the material thickness and electrical characteristics, we can derive the following equation:

$$
\begin{aligned}
& S E(d B)=20 \log \left[\mathrm{e}^{a t} \frac{(1+K)^{2}}{4 K}\left\{1-\left(\frac{K-1}{K+1}\right)^{2} e^{-2 r t}\right\}\right] \\
& =20 \log \left(e^{a t}\right)+20 \log \left\{\frac{(1+K)^{2}}{4 K}\right\} \\
& +20 \log \left\{1-\left(\frac{K-1}{K+1}\right)^{2} e^{-2 r t}\right\}
\end{aligned}
$$

where denotes the ratio between electromagnetic impedance and shield material impedance; $t$, the material thickness; $\gamma$, the propagation constant $(=\alpha+i \beta)$ of the electromagnetic wave; and $\alpha$, the attenuation constant of the electromagnetic wave. In Eq. (2), the first term is called adsorption loss, the second term is called reflection loss, and the third term is called multi-reflection correction. The EMI shield effectiveness is found by the sum of the absorption, the reflection of the electromagnetic wave introduced, and the multireflection between the medium boundaries. The electromagnetic wave can be shielded effectively by utilizing these losses according to the characteristics of the electromagnetic wave to be shielded. That is, when electromagnetic energy arrives at an arbitrary object (shielding material), the travel path of the electromagnetic wave is distributed by three phenomena, namely reflection, transmission, and adsorption. Here, shielding is defined by combining the reflection and the adsorption when a criterion is set within a system. With respect to the actual electromagnetic measurement, the signal that is generated at the antenna on the generation side is called PT, and the signal received by the antenna on the receiving side is called PR. Assuming that a signal on the receiving side, in which a shield is arranged between the generation and the receiving sides using a shielding material, is P'R, we can calculate the effectiveness of the EMI shield by using Eq. (1). Fig. 6 shows this arrangement [12].

In order to measure the electrical conductivity, the electrical characteristics (resistance, R) were measured by a four-terminal method using a KEI-THLEY 580 Micro-Ohmmeter when the orientation angle was $0 / 0,0 / 15,0 / 30,0 / 45$, and $0 / 90$. Then, according to Eq. (3), the volume resistivity $\left(\rho_{\mathrm{o}}\right)$ was calculated, and the relative value of the material was compared.

$$
\rho_{o}=\left(\frac{E}{I_{0}}\right)\left(\frac{A}{t}\right)=\frac{R_{0} A}{t}
$$




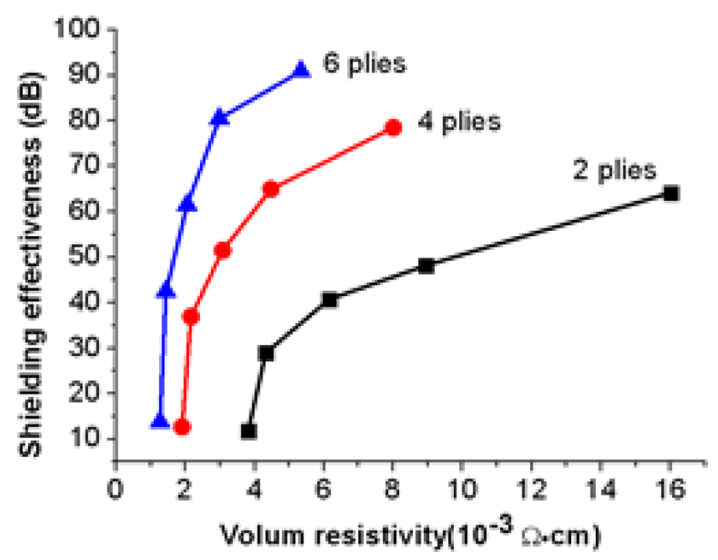

Fig. 7. Average shielding effectiveness as to volume resistivity of carbon fiber reinforced plastic.

\begin{tabular}{lcc}
$\begin{array}{l}\text { Table 1. Volume resistivity as to carbon fiber orientation angle in } \\
2 \text { plies }\end{array}$ \\
$\begin{array}{c}\text { Fiber } \\
\text { orientation }\end{array}$ & $\begin{array}{c}\text { Volume resistivity } \\
(\Omega \bullet \mathrm{cm})\end{array}$ & $\begin{array}{c}\text { Shielding effectiveness } \\
(\mathrm{dB})\end{array}$ \\
\hline $0^{\circ} / 0^{\circ}$ & $3.810 \times 10^{-3}$ & 11.76150 \\
$0^{\circ} / 15^{\circ}$ & $4.320 \times 10^{-3}$ & 28.95386 \\
$0^{\circ} / 30^{\circ}$ & $6.154 \times 10^{-3}$ & 40.75300 \\
$0^{\circ} / 45^{\circ}$ & $8.946 \times 10^{-3}$ & 48.19975 \\
$0^{\circ} / 90^{\circ}$ & $1.601 \times 10^{-2}$ & 64.16224 \\
\hline
\end{tabular}

Table 2. Volume resistivity as to carbon fiber orientation in 4 plies

\begin{tabular}{ccc}
$\begin{array}{c}\text { Fiber } \\
\text { orientation }\end{array}$ & $\begin{array}{c}\text { Volume resistivity } \\
(\Omega \cdot \mathrm{cm})\end{array}$ & $\begin{array}{c}\text { Shielding effectiveness } \\
(\mathrm{dB})\end{array}$ \\
\hline $0^{\circ} / 0^{\circ}$ & $1.905 \times 10^{-3}$ & 12.68298 \\
$0^{\circ} / 15^{\circ}$ & $2.160 \times 10^{-3}$ & 36.92421 \\
$0^{\circ} / 30^{\circ}$ & $3.077 \times 10^{-3}$ & 51.50091 \\
$0^{\circ} / 45^{\circ}$ & $4.473 \times 10^{-3}$ & 64.98028 \\
$0^{\circ} / 90^{\circ}$ & $8.005 \times 10^{-3}$ & 78.49058 \\
\hline
\end{tabular}

Table 3. Volume resistivity as to carbon fiber orientation in 6 plies

\begin{tabular}{ccc}
$\begin{array}{c}\text { Fiber } \\
\text { orientation }\end{array}$ & $\begin{array}{c}\text { Volume resistivity } \\
(\Omega \cdot \mathrm{cm})\end{array}$ & $\begin{array}{c}\text { Shielding effectiveness } \\
(\mathrm{dB})\end{array}$ \\
\hline $0^{\circ} / 0^{\circ}$ & $1.270 \times 10^{-3}$ & 13.80939 \\
$0^{\circ} / 15^{\circ}$ & $1.440 \times 10^{-3}$ & 42.55058 \\
$0^{\circ} / 30^{\circ}$ & $2.051 \times 10^{-3}$ & 61.5261 \\
$0^{\circ} / 45^{\circ}$ & $2.982 \times 10^{-3}$ & 80.51934 \\
$0^{\circ} / 90^{\circ}$ & $5.336 \times 10^{-3}$ & 90.88604 \\
\hline
\end{tabular}

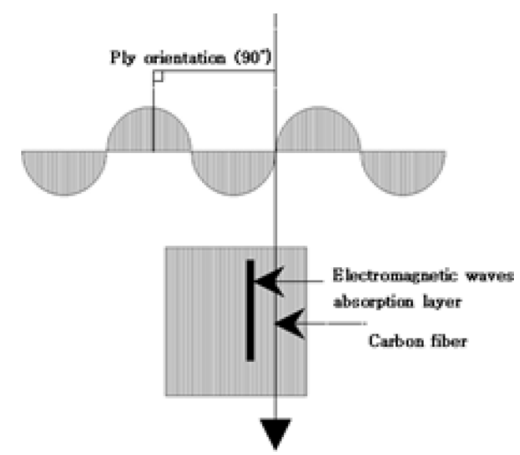

Fig. 8. Shielding effectiveness ofcarbon fiber reinforced plastic with a fiber orientation angle of $90^{\circ}$.

where $\mathrm{E}$ is the voltage $(\mathrm{V})$ applied to the specimen; $I_{o}$, the measured current (A); $R_{o}$, the measured resistance $\mathrm{R} ; \mathrm{t}$, the thickness of the specimen; and A, the contact surface area $\left(\mathrm{cm}^{2}\right)$ of the electrode.

\section{Experiment Results and Discussion}

The CFRP has a low resistance rate of $10^{-2} \sim 10^{-3} \Omega \cdot \mathrm{cm}$ because of the conductivity of the carbon fiber. These electrical characteristics were measured with respect to the volume resistivity of the specimens of 2 plies, 4 plies, and 6 plies by changing the orientation angle, as shown in Fig. 2. Tables 1-3 and Fig. 7 show the characteristics. Theoretically, a specimen that has an orientation angle of $0^{\circ}$ and a low electrical resistance value should have the best reflection effect of an electromagnetic wave. However, since the material used in this test was fiber-reinforced plastic that has thermal and electrical anisotropy, the shielding effectiveness measured as the orientation angle showed that the angle of $90^{\circ}$, which was perpendicular to the electromagnetic wave propagation, had the maximum effectiveness, as shown in Fig. 7. This result was obtained because of the effect of the fiber arrangement in the horizontal direction of the electric field, while considering the conductivity loss. That is, when an electric field and resistance conductor are placed in parallel, a current is generated, which can explain the absorption of electromagnetic waves as a resistance thermal loss [12].

The experiment of the EMI shield was performed to find out the electromagnetic shielding performance according to the number of plies and the orientation angle of the CFRP. The frequency ranged between $500 \mathrm{MHz}$ and $3 \mathrm{GHz}$, and the reference was set as $20 \mathrm{dBmV}$. Figs. 9-13 show the results of the electromagnetic shielding performance based on the changes in the number of plies of the carbon fiber while the orientation angle $\theta$ was constant.

Fig. 9 shows the shielding effectiveness when the orientation angle $\theta$ was $0^{\circ}$. Fig. 9a shows the electromagnetic reflection loss. With respect to the reflection loss based on the number of plies, assuming $100 \%$ in the case of 2 plies on average, there was slight decrease of $6.2 \%$ in the case of 4 plies and $7.7 \%$ in the case of 6 plies. Fig. $9 \mathrm{~b}$ shows the absorption loss of the electromagnetic wave. As shown in the figure, 


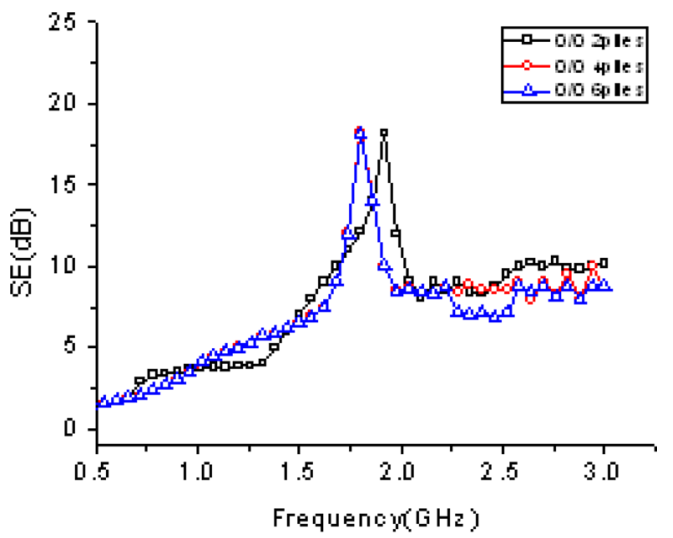

(a)

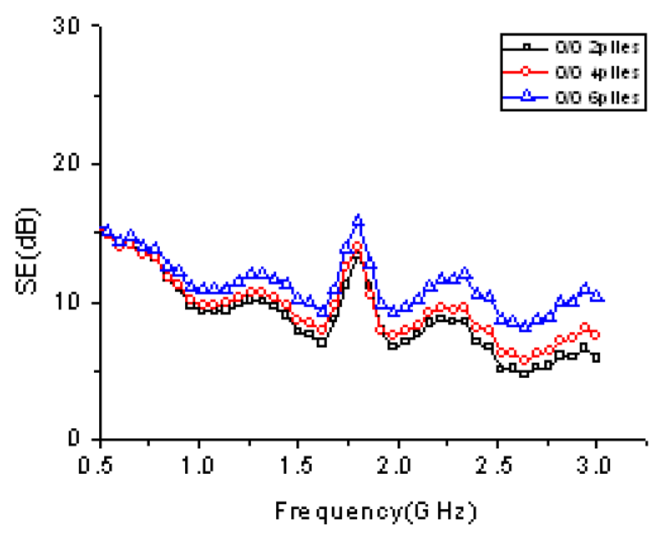

(b) Transmission loss

Fig. 9. Shielding effectiveness (SE) of carbon fiber reinforced plastic according to the number of plies for a fiber orientation angle of $0^{\circ} / 0^{\circ}$. (a) Reflection loss, (b) transmission loss.

as the number of plies increased, the shielding performance increased, while the performance difference showed a slight increase of $7.8 \%$ in the case of 4 plies and $17.4 \%$ in the case of 6 plies, assuming $100 \%$ in the case of 2 plies. According to the results shown in Fig. 9, at an orientation angle $\theta$ of $0^{\circ}$, the reflection loss as to the number of plies had a maximum difference of $6.0 \mathrm{~dB}$ at a peak value according to the number of plies, while the adsorption loss was influenced minimally ( $3 \mathrm{~dB}$ on average).

Fig. 10 shows the shielding effectiveness when the orientation angle $\theta$ was $15^{\circ}$. Fig. 10a shows the electromagnetic reflection loss. As shown in the figure, the reflection loss according to the number of plies decreased by $0.8 \%$ in the case of 4 plies and slightly increased by $1.2 \%$ in the case of 6 plies, assuming $100 \%$ in the case of 2 plies. Fig. 10b shows the electromagnetic absorption loss. The shielding performance increased with an increase in the number of plies. The performance difference increased by $27.5 \%$ in the case of 4 plies and $46.9 \%$ in the case of 6 plies, assuming $100 \%$ in the case of 2 piles. According to the results shown in Fig. 10, when the orientation angle $\theta$ was $15^{\circ}$, the effect on the reflection loss was maximum, i.e., $1.5 \mathrm{~dB}$, at the peak value showing a slight difference. The effect on the adsorption loss, which is $13 \mathrm{~dB}$ on average, increased according

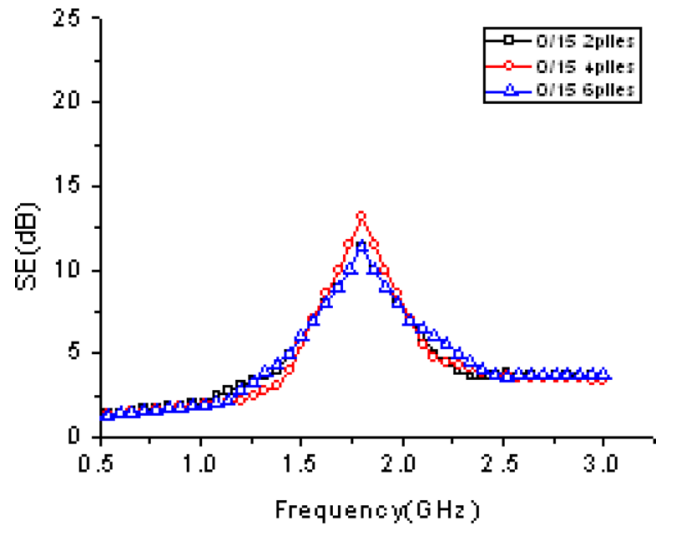

(a)

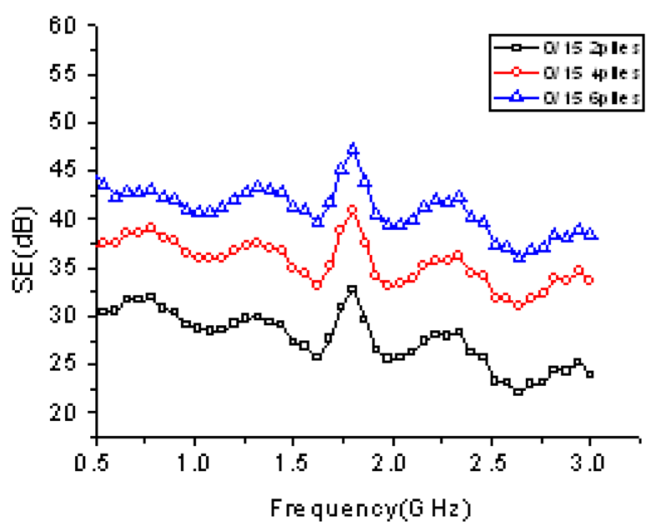

(b)

Fig. 10. Shielding effectiveness (SE) of carbon fiber reinforced plastic according to the number of plies for a fiber orientation angle of $0^{\circ} / 15^{\circ}$. (a) Reflection loss, (b) transmission loss.

to the number of plies.

Fig. 11 shows the shielding effectiveness when the orientation angle $\theta$ was $30^{\circ}$. Fig. 11a shows the electromagnetic reflection loss. As shown in the figure, the reflection loss according to the number of plies decreased by $24.9 \%$ in the case of 4 plies and $34.7 \%$ in the case of 6 plies, assuming $100 \%$ in the case of 2 plies. Fig. $11 \mathrm{~b}$ shows the electromagnetic absorption loss. The shielding performance increased with an increase in the number of plies. The performance difference increased by $26.3 \%$ in the case of 4 plies and $50.9 \%$ in the case of 6 plies, assuming $100 \%$ in the case of 2 piles. According to the results shown in Fig. 11, when the orientation angle $\theta$ was $30^{\circ}$, the effect on the reflection loss showed a maximum difference of $6.2 \mathrm{~dB}$ at the peak value. The effect on the adsorption loss, which is $21 \mathrm{~dB}$ on average, increased according to the number of plies.

Fig. 12 shows the shielding effectiveness when the orientation angle $\theta$ was $45^{\circ}$. Fig. 12a shows the electromagnetic reflection loss. As shown in the figure, the reflection loss according to the number of plies decreased by $0.8 \%$ in the case of 4 plies and $5.7 \%$ in the case of 6 plies, assuming $100 \%$ in the case of 2 plies. Fig. 12b shows the electromagnetic absorption loss. The shielding performance increased with an 


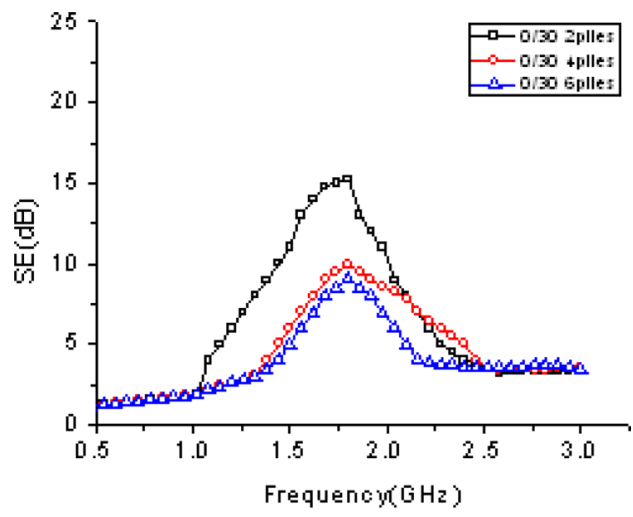

(a)

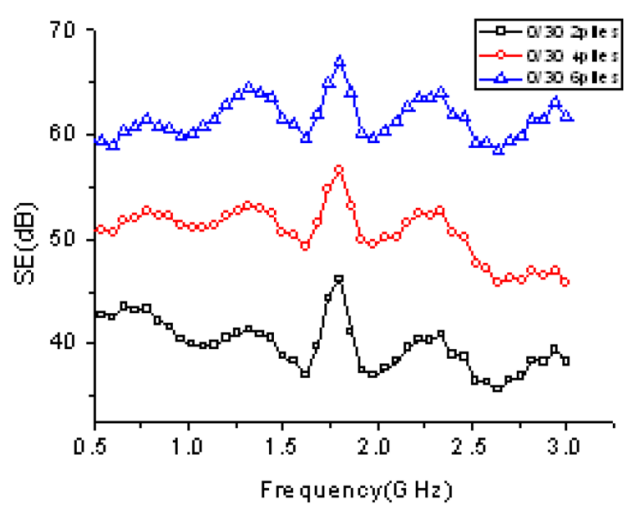

(b)

Fig. 11. Shielding effectiveness (SE) of carbon fiber reinforced plastic according to the number of plies for a fiber orientation angle of $0^{\circ} / 30^{\circ}$. (a) Reflection loss, (b) transmission loss.

increase in the number of plies. The performance difference increased by $34.8 \%$ in the case of 4 plies and $67.0 \%$ in the case of 6 plies, assuming $100 \%$ in the case of 2 piles. According to the results shown in Fig. 12, when the orientation angle $\theta$ was $45^{\circ}$, the effect on the reflection loss showed a maximum difference of $2.6 \mathrm{~dB}$ at the peak value. The effect on the adsorption loss, which is $32.3 \mathrm{~dB}$ on average, increased according to the number of plies.

Fig. 13 shows the shielding effectiveness when the orientation angle $\theta$ was $90^{\circ}$. Fig. 13a shows the electromagnetic reflection loss. As shown in the figure, the reflection loss according to the number of plies decreased by $16.4 \%$ in the case of 4 plies and $12.1 \%$ in the case of 6 plies, assuming $100 \%$ in the case of 2 plies. Fig. 13b shows the electromagnetic absorption loss. The shielding performance increased with an increase in the number of plies. The performance difference increased by $22.3 \%$ in the case of 4 plies and $41.6 \%$ in the case of 6 plies, assuming $100 \%$ in the case of 2 piles. According to the results shown in Fig. 13, when the orientation angle $\theta$ was $90^{\circ}$, the effect on the reflection loss showed a maximum difference of $4.3 \mathrm{~dB}$ at the peak value. The effect on the adsorption loss, which is $32.3 \mathrm{~dB}$ on average, increased according to the number of plies.

Summarizing the results of Figs. 9-13, we found that as

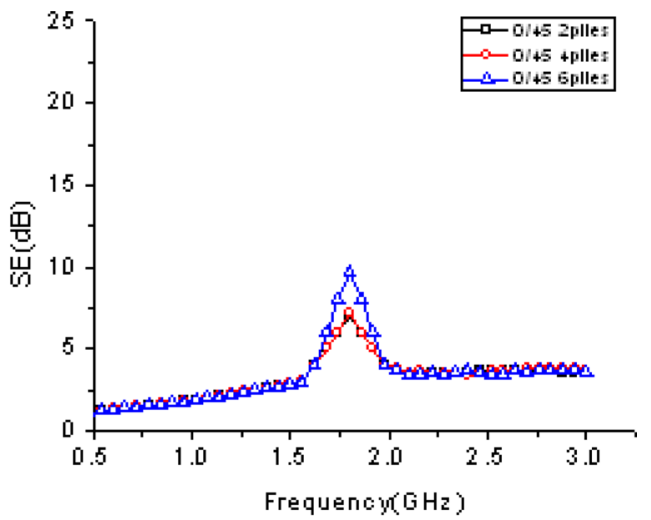

(a)

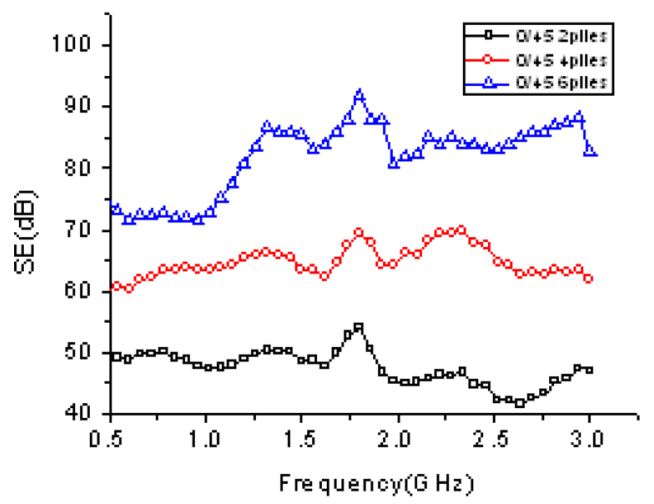

(b)

Fig. 12. Shielding effectiveness (SE) of carbon fiber reinforced plastic according to the number of plies for a fiber orientation angle of $0^{\circ} / 45^{\circ}$. (a) Reflection loss, (b) transmission loss.

the orientation angle $\theta$ increased, the variance of the EMI shield performance also increased according to the number of plies. With respect to the reflection loss, a certain difference was observed according to the number of plies, but overall, the effect was minimal. With respect to the adsorption loss, the orientation angle increased as observed. This was due to a mixture of the increasing effect of the electromagnetic adsorption according to the orientation angle and the increasing areas of electromagnetic adsorption according to the number of plies. Further, at the angles of $0^{\circ}$ and $15^{\circ}$, as the frequency increased, the adsorption loss tended to decrease, while all the frequency bandwidths showed a similar result at the angles of $30^{\circ}$ and $45^{\circ}$. In the case of $90^{\circ}$, as the frequency increased, the absorption loss tended to increase. This phenomenon can be explained as follows: when the orientation angle increased, it became close to the electromagnetic propagation direction, which was $90^{\circ}$, thereby increasing the amount of adsorption loss.

Fig. 14 shows the EMI shield performance result when the number of plies was 2. Fig. 14a shows the electromagnetic reflection loss. The effect on the reflection loss according to the orientation angle decreased by $39.5 \%$ at $0 / 15,17.8 \%$ at $0 / 30$, $56.5 \%$ at $0 / 45$, and $50.3 \%$ at $0 / 90$, assuming $100 \%$ at $0 / 0$. Fig. $14 \mathrm{~b}$ shows the adsorption loss of the electromagnetic wave ac- 


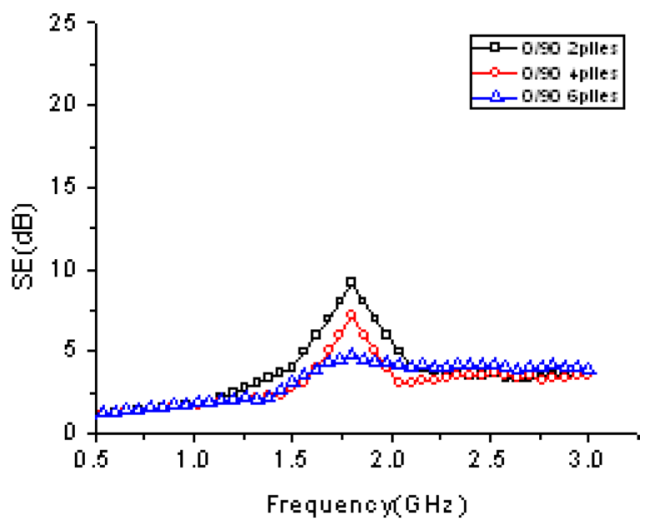

(a)

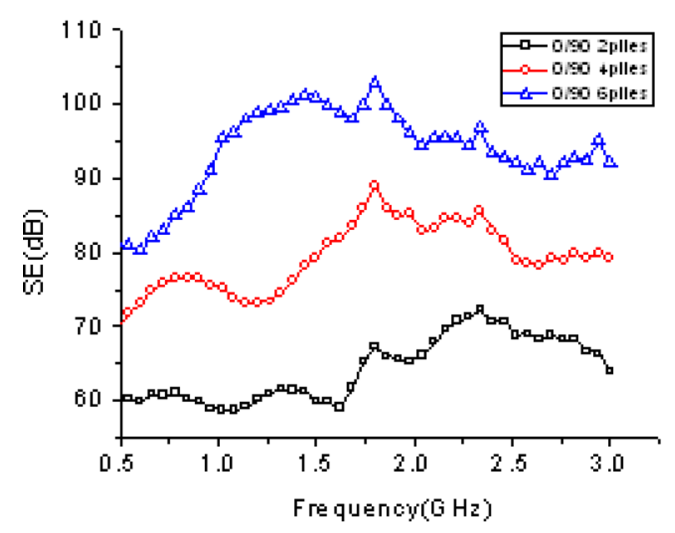

(b)

Fig. 13. Shielding effectiveness (SE) of carbon fiber reinforced plastic according to the number of plies for a fiber orientation angle of $0^{\circ} / 90^{\circ}$. (a) Reflection loss, (b) transmission loss.

cording to the orientation angle, showing an increasing trend of $146.1 \%$ at $0 / 15,246.5 \%$ at $0 / 30,309.7 \%$ at $0 / 45$, and $445.5 \%$ at $0 / 90$, assuming $100 \%$ at $0 / 0$. The results shown in Fig. 14 revealed that the effect on the reflection loss in the case of 2 plies according to the orientation angle was a maximum of $2.9 \mathrm{~dB}$ at the peak value, and the effect on the adsorption loss increased by $52.4 \mathrm{~dB}$ on average.

Fig. 15 shows the EMI shield performance result when the number of plies was 4 . Fig. 15a shows the electromagnetic reflection loss. The effect on the reflection loss according to the orientation angle decreased by $37.01 \%$ at $0 / 15,35.3 \%$ at $0 / 30$, $54.1 \%$ at $0 / 45$, and $56.4 \%$ at $0 / 90$, assuming $100 \%$ at $0 / 0$. Fig. $15 \mathrm{~b}$ shows the adsorption loss of the electromagnetic wave according to the orientation angle, showing an increasing trend of $191.1 \%$ at $0 / 15,306.0 \%$ at $0 / 30,412.4 \%$ at $0 / 45$, and $518.9 \%$ at $0 / 90$, assuming $100 \%$ at $0 / 0$. The results shown in Fig. 15 revealed that the effect on the reflection loss in the case of 4 plies according to the orientation angle was a maximum of $11.0 \mathrm{~dB}$ at the peak value. The effect on the adsorption loss also increased by $65.8 \mathrm{~dB}$ on average.

Fig. 16 shows the EMI shield performance result when the number of plies was 6. Fig. 16a shows the electromagnetic reflection loss. The effect on the reflection loss according to the orientation angle decreased by $33.5 \%$ at $0 / 15,41.8 \%$ at $0 / 30$,

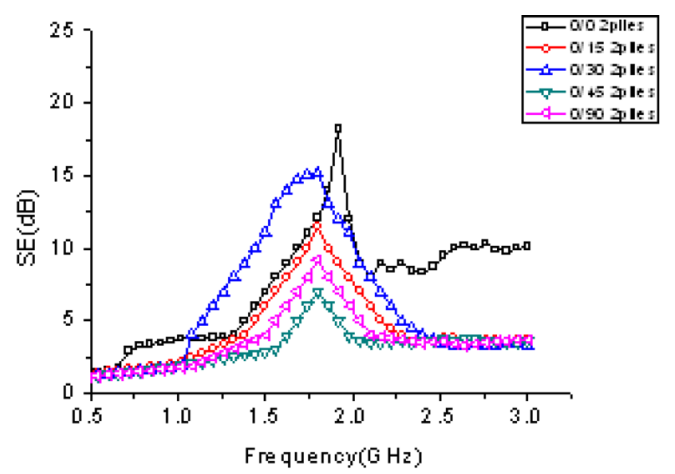

(a)

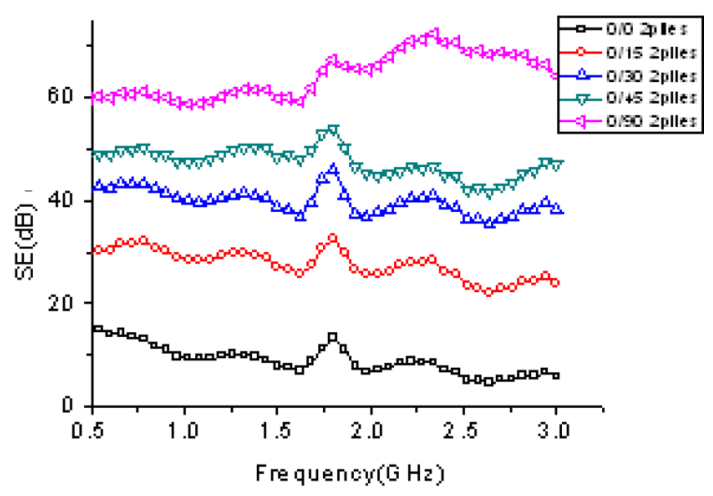

(b)

Fig. 14. Shielding effectiveness (SE) of carbon fiber reinforced plastic according to the fiber orientation angle in the case of 2 plies. (a) Reflection loss, (b) transmission loss.

$50.2 \%$ at $0 / 45$, and $52.7 \%$ at $0 / 90$, assuming $100 \%$ at $0 / 0$. Fig. $16 \mathrm{~b}$ shows the adsorption loss of the electromagnetic wave according to the orientation angle, showing an increasing trend of $208.3 \%$ at $0 / 15,345.8 \%$ at $0 / 30,483.3 \%$ at $0 / 45$, and $558.5 \%$ at $0 / 90$, assuming $100 \%$ at $0 / 0$. The results shown in Fig. 16 revealed the effect on the reflection loss in the case of 6 plies according to the orientation angle, which was a maximum of $2.9 \mathrm{~dB}$ at the peak value. The effect on the adsorption loss also increased by $77 \mathrm{~dB}$ on average.

Summarizing the results of Figs. 14-16, we found that when the number of plies was constant, the EMI shield performance increased with an increase in the orientation angle of the carbon fiber. The reflection loss showed a certain difference according to the orientation angle, although the overall effect was minimal. Further, as the orientation angle increased, the reflection loss tended to decrease. This phenomenon can be explained as follows: As the orientation angle increased, it became close to $90^{\circ}$, which was perpendicular to the electromagnetic propagation direction. This thereby increased the adsorption loss of the electromagnetic wave while decreasing the reflection loss. Furthermore, most of the specimens showed an increasing trend of the EMI shield performance around $1.8 \mathrm{GHz}$. This was due to the characteristics of the carbon fiber with respect to the electromagnetic wave. Finally, the adsorption loss showed an increasing trend when the changes in the orientation angle became relatively large. 


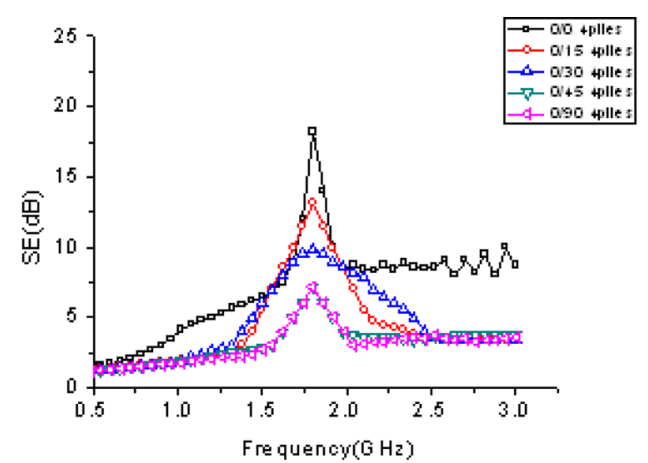

(a)

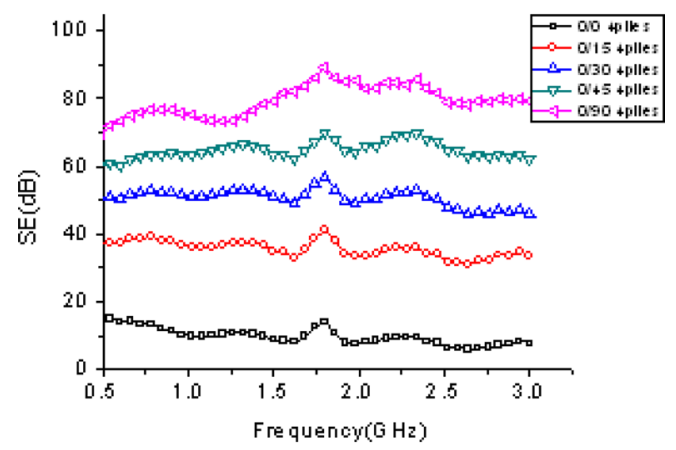

(b)

Fig. 15. Shielding effectiveness (SE) of carbon fiber reinforced plastic according to the fiber orientation angle in the case of 4 plies. (a) Reflection loss, (b) transmission loss.

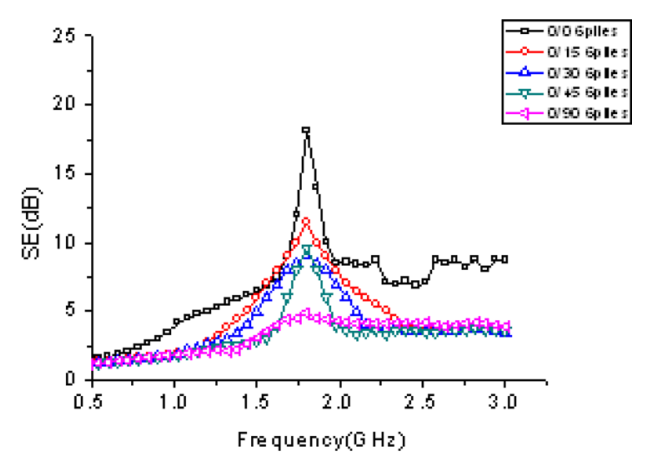

(a)

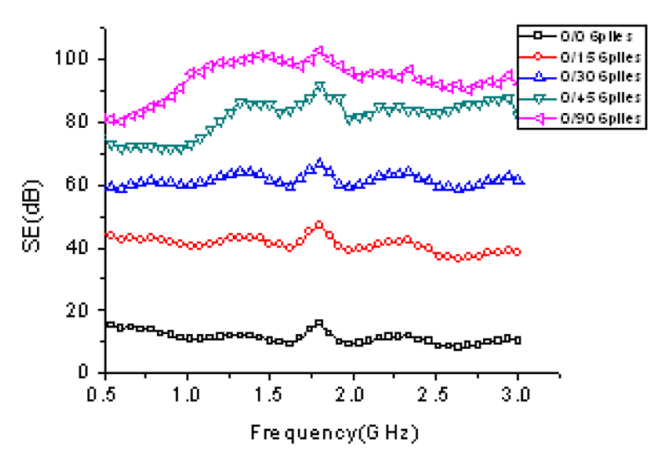

(b)

Fig. 16. Shielding effectiveness (SE) of carbon fiber reinforced plastic according to the fiber orientation angle in the case of 6 plies. (a) Reflection loss, (b) transmission loss.

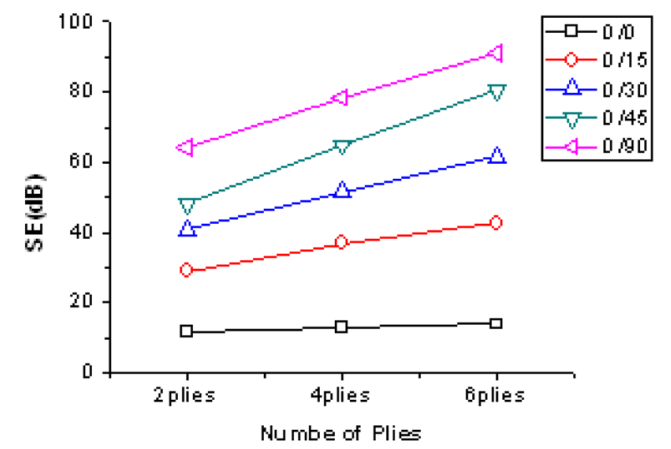

(a)

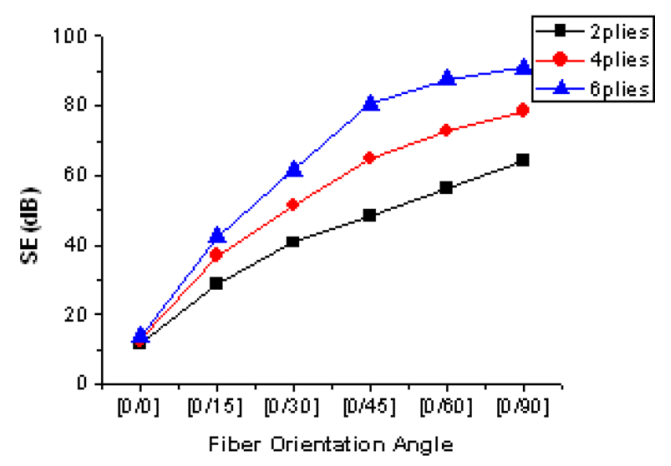

(b)

Fig. 17. Average variation of SE according to the fiber orientation angle and the number of plies. (a) SE according to the number of plies, (b) SE according to the fiber orientation angle.

The changes increased in the order of 2 plies, 4 plies, and 6 plies, which showed the largest increase. This indicates that as the number of plies increased, the adsorption region of the electromagnetic waves also increased.

Fig. 17 shows the average variations of the EMI shielding effectiveness measured for each specimen. As shown in the figure, the EMI shield performance increased when the orientation angle and the number of plies increased. Further, as the orientation angle increased, the gradient of the graph also increased. That is, when the orientation angle was close to $90^{\circ}$, which was the electromagnetic wave propagation angle, the shielding amount also increased. This result implied that the EMI shield performance of CFRP was influenced more by the orientation angle than the number of plies.

\section{Conclusions}

In this study, we studied the EMI shield performance according to the orientation of the lamination of CRFP and the number of plies. We derived the following results:

It was verified that as the number of plies of the CFRP increased, the EMI shield performance increased. This was due to the effect of the large absorption area for the electromagnetic waves.

It was verified that as the orientation angle of the CFRP increased, the EMI shield performance increased. This indicates that as the orientation angle increased, the angle became close 
to $90^{\circ}$, which was perpendicular to the electromagnetic wave propagation direction. Therefore, the absorption loss of the electromagnetic wave increased.

It was verified that as the number of plies and the orientation angle increased, the reflection loss tended to decrease slightly while the absorption loss increased. This was due to the large absorption region of the electromagnetic wave (the orientation angle and the number of plies).

Irrespective of the lamination direction and the number of plies, most specimens showed an increasing performance of the EMI shield at a frequency of around $1.8 \mathrm{GHz}$. This can be explained by the characteristics of the electromagnetic wave of the carbon fiber.

The orientation angle influenced the effectiveness of the EMI shielding more than the number of CFRP plies. These results suggested that when designing electromagnetic shield materials and components using the carbon fiber, the frequency bandwidth required for the EMI shield and the shield performance must be satisfied by adjusting the orientation angle and the number of plies geometrically.

\section{Acknowledgements}

This research was financially supported by the Ministry of Education, Science Technology (MEST) and National Research Foundation of Korea (NRF) through the Human Resource Training Project for Regional Innovation (No. 2012H1B8A2026147) and (No. 2013R1A1A2062899).

\section{References}

[1] Jung HJ, Choi HS, Kim EK. A comparative study of the shielding performance of uniforms using electromagnetic wave shielding materials currently on the market for workers at Korea Railroad Corporation. J Korean Soc Costume, 60, 23 (2010).

[2] Johnson CC, Guy AW. In: Osepchuk JM, IEEE Committee on Man and Radiation, eds. Biological Effects of Electromagnetic Radia- tion, IEEE Press, New York, NY, 43 (1983).

[3] Luo X, Chung DDL. Electromagnetic interference shielding using continuous carbon-fiber carbon-matrix and polymer-matrix composites. Composites B, 30, 227 (1999). http://dx.doi.org/10.1016/ S1359-8368(98)00065-1.

[4] Park JG, Louis J, Cheng Q, Bao J, Smithyman J, Liang R, Wang B, Zhang C, Brooks JS, Kramer L, Fanchasis P, Dorough D. Electromagnetic interference shielding properties of carbon nanotube buckypaper composites. Nanotechnology, 20, 415702 (2009). http://dx.doi.org/10.1088/0957-4484/20/41/415702.

[5] Kim JG, Chung CH, Lee YS. The effect of crystallization by heat treatment on electromagnetic interference shielding efficiency of carbon fibers. Appl Chem Eng, 22, 138 (2011).

[6] Dewhurst RJ, Edwards C, McKie ADW, Palmer SB. Estimation of the thickness of thin metal sheet using laser generated ultrasound. Appl Phys Lett, 51, 1066 (1987). http://dx.doi. org/10.1063/1.98793.

[7] Al-Saleh MH, Sundararaj U. Electromagnetic interference shielding mechanisms of CNT/polymer composites. Carbon, 47, 1738 (2009). http://dx.doi.org/10.1016/j.carbon.2009.02.030.

[8] Chung DDL. Comparison of submicron-diameter carbon filaments and conventional carbon fibers as fillers in composite materials. Carbon, 39, 1119 (2001). http://dx.doi.org/10.1016/S00086223(00)00314-6.

[9] Chung DDL. Electromagnetic interference shielding effectiveness of carbon materials. Carbon, 39, 279 (2001). http://dx.doi. org/10.1016/S0008-6223(00)00184-6.

[10] Kim JB, Kim CG. Study on design and performance of microwave absorbers of carbon nanotube composite laminates. J Korean Soc Compos Mater, 24, 38 (2011). http://dx.doi.org/10.7234/ kscm.2011.24.2.038.

[11] Han GY, Song DH, Bae JS, Ahn DG. A study on electromagnetic interference shielding effectiveness of the aluminum film, conductive fabric, and nano carbon black/carbon fiber reinforced composites. J Korean Soc Manuf Process Eng, 7, 10 (2008).

[12] Hong MS, Bae KM, Lee HS, Park SJ, An KH, Kang SJ, Kim BJ. Electromagnetic interference shielding behaviors of electrolysis nickel-loaded carbon fibers-reinforced epoxy matrix composites. Appl Chem Eng, 22, 672 (2011). 